\title{
Ground effects of space weather investigated by the surface impedance
}

\author{
Risto Pirjola ${ }^{1,2}$, David Boteler ${ }^{2}$, and Larisa Trichtchenko ${ }^{2}$ \\ ${ }^{1}$ Finnish Meteorological Institute, P. O. Box 503, FI-00101 Helsinki, Finland \\ ${ }^{2}$ Natural Resources Canada, Geological Survey of Canada, Geomagnetic Laboratory, \\ 7 Observatory Crescent, Ottawa, Ontario, K1A 0Y3, Canada
}

(Received February 1, 2008; Revised September 1, 2008; Accepted September 26, 2008; Online published February 18, 2009)

\begin{abstract}
The objective of this paper is to provide a discussion of the surface impedance applicable in connection with studies of geomagnetically induced currents (GIC) in technological systems. This viewpoint means that the surface impedance is regarded as a tool to determine the horizontal (geo)electric field at the Earth's surface, which is the key quantity for GIC. Thus the approach is different from the traditional magnetotelluric viewpoint. The definition of the surface impedance usually involves wavenumber-frequency-domain fields, so inverse Fourier transforming the expression of the electric field in terms of the surface impedance and the geomagnetic field results in convolution integrals in the time and space domains. The frequency-dependent surface impedance has a high-pass filter character whereas the corresponding transfer function between the electric field and the time derivative of the magnetic field is of a low-pass filter type. The relative change of the latter transfer function with frequency is usually smaller than that of the surface impedance, which indicates that the geoelectric field is closer to the time derivative than to the magnetic field itself. An investigation of the surface impedance defined by the space-domain electric and magnetic components indicates that the largest electric fields are not always achieved by the plane wave assumption, which is sometimes regarded as an extreme case for GIC. It is also concluded in this paper that it is often possible to apply the plane wave relation locally between the surface electric and magnetic fields. The absolute value of the surface impedance decreases with an increasing wavenumber although the maximum may also be at a non-zero value of the wavenumber. The imaginary part of the surface impedance usually much exceeds the real part.
\end{abstract}

Key words: Geoelectric field, geomagnetic field, geomagnetically induced current, GIC, space weather, plane wave, convolution.

\section{Introduction}

"Space Weather" originating from solar activity is an important research subject in a modern society because vital space-borne and ground-based technological systems may suffer from problems during space storms (e.g. Lanzerotti $e t$ al., 1999). At the Earth's surface space weather manifests itself as geomagnetically induced currents (GIC) in largescale conductor networks, such as electric power transmission grids, oil and gas pipelines, telecommunication cables and railway systems (e.g. Boteler et al., 1998). The key quantity for GIC is the horizontal geoelectric field induced by a geomagnetic disturbance or storm at the Earth's surface. The transfer function between the geoelectric and geomagnetic fields is known as the surface impedance (e.g. Kaufman and Keller, 1981).

The concept of the surface impedance, usually denoted by $Z$, (or of the inductive response function, or of the complex depth or of the apparent resistivity directly derivable from $Z$ ) is included implicitly or explicitly in magnetotelluric research since its beginning (e.g. Cagniard, 1953; Wait, 1954; Schmucker, 1970a, b; Schmucker and Jankowski, 1972; Weidelt, 1972; Weaver, 1973; Thomson

Copyright (c) The Society of Geomagnetism and Earth, Planetary and Space Sciences (SGEPSS); The Seismological Society of Japan; The Volcanological Society of Japan; The Geodetic Society of Japan; The Japanese Society for Planetary Sciences; TERRAPUB and Weaver, 1975; Dmitriev and Berdichevsky, 1979; Wait, 1980; Avdeev et al., 1989; Weaver and Agarwal, 1993; Dmitriev and Berdichevsky, 2002; and many others). The objective of this paper is not to repeat the well-known applications of the use of the surface impedance to investigations of the Earth's conductivity structure but to explore, demonstrate and summarise the properties of the surface impedance paying special attention to its utilisation in the calculation of the geoelectric field for space weather purposes. A usual practical problem is to determine the electric field at the Earth's surface from ground-based magnetic recordings, and the surface impedance plays the key role then (e.g. Boteler, 1994; Viljanen et al., 2004).

Similarly to the electric and magnetic fields, the surface impedance is affected primarily by ionosphericmagnetospheric currents and secondarily by the Earth's conductivity structure. In general, $Z$ is a $2 \times 2$ tensor but in many applications it can simply be defined as the scalar ratio of the perpendicular electric and magnetic components. In order that the unit of the impedance is 'ohm' $[\Omega]$, we include the vacuum permeability $\mu_{0}(=$ $4 \pi \cdot 10^{-7} \mathrm{~V} \mathrm{~s} \mathrm{~A}^{-1} \mathrm{~m}^{-1}$ ) in the definition of $Z$, which is based on the magnetic flux density field $\mathbf{B}$, instead of the magnetic field intensity $\mathbf{H}$, in this paper. In the case of the electric $y$ and magnetic $x$ component a minus sign is also added for convenience in the definition of $Z$. 
The surface impedance is usually, but not necessarily, determined from the electric and magnetic fields in the frequency-wavenumber domain. This means that the electric and magnetic data have to be Fourier transformed from the time to the frequency and from one or two horizontal space coordinates to one or two wavenumbers. For simplicity, in this paper, we only consider one horizontal coordinate and thus one wavenumber. Inverse Fourier transforms from the frequency to the time and from the wavenumber to the space domain result in convolution integrals.

The special situation with electric and magnetic fields that are independent of the space coordinates at the Earth's surface is called the "plane wave case", which is the most important in practice in connection with the surface impedance. It corresponds to a zero wavenumber. Important conclusions about the relation between the electric and magnetic fields in the time domain can be drawn from the behaviour of $Z$ in the frequency domain. Calculating the surface impedance from electric and magnetic fields dependent on a space coordinate at the Earth's surface indicates that $Z$ varies much from site to site and its absolute value may be larger or smaller than in the plane wave case, which thus does not constitute any extreme case.

The paper is organised by presenting the theory and the equations in Section 2, and Section 3 is devoted to numerical examples, which are chosen regarding applications to geoelectric and GIC modelling.

\section{Theory}

\subsection{Definition of the surface impedance}

The (scalar) surface impedance $Z$ is defined by

$$
Z=-\mu_{0} \frac{E_{y}}{B_{x}}
$$

where $E_{y}$ and magnetic $B_{x}$ are perpendicular horizontal electric and magnetic components at the Earth's surface. The usual choice of the Cartesian $x y z$ coordinate system in geoelectromagnetic research (as in this paper) implies that the Earth's surface is the $x y$ plane and the $x, y$ and $z$ axes point northwards, eastwards and downwards, respectively. Equation (1) provides a general definition of the surface impedance independently of the domains (time, frequency, space, wavenumber) in which $E_{y}$ and $B_{x}$ are considered. Note that, in the frequency and wavenumber domains, $E_{y}$ and $B_{x}$ are generally complex-valued quantities making $Z$ complex, too. A recursive formula for the surface impedance in the frequency and wavenumber domains can be derived for a layered Earth by using the continuity conditions of the electric and magnetic fields at the layer boundaries (e.g. Wait, 1981, pp. 43-55). This formula is used in the numerical examples presented in Section 3.

\subsection{Plane wave case}

We now assume that the Earth has a layered structure and that the primary electromagnetic field due to ionospheric and magnetospheric currents is a plane wave propagating vertically downwards. Then both the secondary reflected field in the air and the fields in the Earth layers also propagate vertically and no dependence on $x$ or $y$ exists. This is called the "plane wave case", and the surface impedance characterising the Earth's conductivity structure only de- pends on the angular frequency $\omega$ (provided that $E_{y}$ and $B_{x}$ are Fourier transformed from the time $(t)$ to the frequency $(\omega)$ domain). Thus

$$
E_{y}(\omega)=-\frac{1}{\mu_{0}} Z_{\mathrm{p}}(\omega) B_{x}(\omega)
$$

where the subscript ' $p$ ' refers to 'plane wave' and $Z_{p}(\omega)$ is the "plane wave surface impedance". Possible violations of the plane wave assumption resulting in so-called source effects have caused a lot of discussion in connection with geoelectromagnetic induction and magnetotelluric studies of the Earth (e.g. Wait, 1954; Mareschal, 1986; Osipova et al., 1989; Viljanen et al., 1993; Varentsov et al., 2003; Sokolova et al., 2007). In general, the farther away the primary currents the better the plane wave assumption is satisfied, which means in practice that the source effect problem becomes significant in auroral and equatorial electrojet regions. As expressed more precisely, neglecting source effects requires that the horizontal variation of the fields should be small in a distance equal to the skin depth in the Earth. This means that large frequencies, i.e. short periods, and highly-conducting Earth structures are more favourable for the plane wave assumption. To quantify this statement, we consider a characteristic length for horizontal variations of the fields to be about $200 \mathrm{~km}$. Then the skin depth in a uniform Earth having a conductivity of $10^{-3} \Omega^{-1} \mathrm{~m}^{-1}$ is small enough for the plane wave approximation to be acceptable if the period is roughly one to two minutes or less. For conductivities of $10^{-1} \Omega^{-1} \mathrm{~m}^{-1}$ and of $10^{-5} \Omega^{-1} \mathrm{~m}^{-1}$, the periods have to be less than about two hours and a second, respectively, to make the skin depth sufficiently small for the plane wave assumption. As discussed e.g. by Dmitriev and Berdichevsky (1979) and by Wait (1980) and implicitly already shown by Wait (1954), a linear spatial variation of the field does not prevent the application of the plane wave formulas. Further discussions of the subject are, however, outside the scope of this paper.

Denoting the time derivative of $B_{x}(t)$ by $g(t)$ (= $\left.d B_{x}(t) / d t\right)$ and using the relation $g(\omega)=i \omega B_{x}(\omega)$, Eq. (2) may be expressed as

$$
E_{y}(\omega)=-\frac{Z_{\mathrm{p}}(\omega)}{i \omega \mu_{0}} g(\omega)
$$

Equations (2) and (3) can be inverse Fourier transformed to obtain the relations between the electric and magnetic fields in the time domain. To get quantitatively correct results, the exact forms of the Fourier transform and of its inverse to be used have to be defined clearly, so we use the following conventions

$$
\begin{aligned}
& F(\omega)=\frac{1}{\sqrt{2 \pi}} \int_{-\infty}^{\infty} F(t) e^{-i \omega t} d t \\
& F(t)=\frac{1}{\sqrt{2 \pi}} \int_{-\infty}^{\infty} F(\omega) e^{i \omega t} d \omega
\end{aligned}
$$

where $[F(t), F(\omega)]$ is the time-frequency Fourier transform pair of any quantity. (Mathematical items including the Fourier transform, the convolution theorem and the Dirac delta function are summarised for geoelectromagnetic purposes e.g. by Pirjola (1982, appendix A).) By applying the 
convolution theorem, Eqs. (2) and (3) together with formula (5) give

$$
\begin{aligned}
E_{y}(t) & =-\frac{1}{\mu_{0} \sqrt{2 \pi}} \int_{-\infty}^{\infty} Z_{\mathrm{p}}(t-u) B_{x}(u) d u \\
& =-\frac{1}{\mu_{0} \sqrt{2 \pi}} \int_{-\infty}^{\infty} Z_{\mathrm{p}}(u) B_{x}(t-u) d u \\
E_{y}(t) & =-\frac{1}{\mu_{0} \sqrt{2 \pi}} \int_{-\infty}^{\infty} C_{\mathrm{p}}(t-u) g(u) d u \\
& =-\frac{1}{\mu_{0} \sqrt{2 \pi}} \int_{-\infty}^{\infty} C_{\mathrm{p}}(u) g(t-u) d u
\end{aligned}
$$

The functions $Z_{\mathrm{p}}(t)$ and $C_{\mathrm{p}}(t)$ are obtained from $Z_{\mathrm{p}}(\omega)$ and $Z_{\mathrm{p}}(\omega) / i \omega$ by using Eq. (5). Consequently, $Z_{\mathrm{p}}(t)$ is the time derivative of $C_{\mathrm{p}}(t)$. The integrals in formulas (6) and (7) extend from $-\infty$ to $+\infty$ indicating that the value of the electric field at a given time $t$ is in principle affected by the magnetic field at all times in the past and in the future. However, Eqs. (6) and (7) actually satisfy the causality, i.e. only past values of the magnetic field have an influence, which mathematically means that $Z_{\mathrm{p}}(t)$ and $C_{\mathrm{p}}(t)$ are zero for negative arguments.

If $Z_{\mathrm{p}}(t)$ is proportional to the Dirac delta function $\delta(t)$ Eq. (6) shows that $E_{y}(t)$ is proportional to $B_{x}(t)$, and similarly based on Eq. (7), $E_{y}(t)$ is proportional to $g(t)$ if $C_{\mathrm{p}}(t)$ is proportional to $\delta(t)$. In the frequency domain, these conditions are equivalent to $Z_{\mathrm{p}}(\omega)$ independent of $\omega$ and to $Z_{\mathrm{p}}(\omega)$ linearly dependent on $\omega$, respectively. The particular conclusions can be drawn either from Eq. (4) with $F(t)$ proportional to $\delta(t)$ (and noting that the Fourier transform of $C_{\mathrm{p}}(t)$ equals $Z_{\mathrm{p}}(\omega) / i \omega$, which is independent of $\omega$ when $Z_{\mathrm{p}}(\omega)$ is proportional to $\omega$ ) or directly from Eqs. (2) and (3). Since the electric and magnetic fields are coupled by Faraday's law of induction involving the time derivative of the magnetic field the first guess might be that $E_{y}(t)$ and $g(t)$ would be proportional. The issue is investigated by using certain layered Earth models in Section 3. The assumption of a uniform Earth (Section 2.6) enables a closed-form integral relation between $E_{y}(t)$ and $g(t)$, which shows that the former is strongly related to the latter but a simple proportionality does not exist. It is worth emphasising here that, at least to the first approximation, the process of the electric field driving GIC in a technological network is a dc process (Lehtinen and Pirjola, 1985; Pulkkinen et al., 2001). Therefore the time behaviour of GIC is the same as that of the electric field, and so the electric field may be replaced by GIC in the conclusions drawn about the relations between the electric field and the magnetic field or its time derivative in the time domain. Trichtchenko and Boteler (2007) present an example in which the electric field and GIC are roughly proportional to the magnetic time derivative and another example in which a close relation to the magnetic field exists. An obvious explanation is that the two sites for these observations are located at different Earth conductivity structures.

\subsection{Surface impedance in the space domain}

Let us consider electric and magnetic fields that have been Fourier transformed from the time domain into the frequency domain and consider a single angular frequency $\omega$. We assume that the only space coordinates on which the fields depend are $x$ and $z$ (a two-dimensional case). Maxwell's equations then lead to two separate sets of equations, one including the electric $E_{y}$ and magnetic $B_{x}$ and $B_{z}$ components and the other including the electric $E_{x}$ and $E_{z}$ and magnetic $B_{y}$ components. We adopt the former, i.e. the $E$ polarisation case. Such an electromagnetic field is produced by an infinitely long line or sheet current simulating an ionospheric electrojet and located parallel to the $y$ axis above a layered Earth and having the (implicit) time dependence $e^{i \omega t}$. Following the general equation (1), the surface impedance denoted by $Z_{\mathrm{s}}$ is now a function of $x$ and $\omega$ and is given by

$$
Z_{\mathrm{s}}=Z_{\mathrm{s}}(x, \omega)=-\mu_{0} \frac{E_{y}(x, \omega)}{B_{x}(x, \omega)}
$$

The surface impedance, of course, also depends on the Earth's conductivity structure, not expressed explicitly in formula (8).

\subsection{Surface impedance in the wavenumber domain}

We continue to consider the $E$ polarisation case similar to that discussed in Section 2.3, i.e. the components $E_{y}$ and $B_{x}$ are functions of $x$ and $\omega$ at the Earth's surface. The Fourier transform and the inverse Fourier transform between the space $(x)$ and wavenumber $(b)$ domains are defined similarly to Eqs. (4) and (5) with $t$ and $\omega$ replaced by $x$ and $b$, respectively. Based on the general Eq. (1), the surface impedance $Z_{\mathrm{w}}$ in the wavenumber domain is

$$
Z_{\mathrm{w}}=Z_{\mathrm{w}}(b, \omega)=-\mu_{0} \frac{E_{y}(b, \omega)}{B_{x}(b, \omega)}
$$

where $E_{y}(b, \omega)$ and $B_{x}(b, \omega)$ are obtained by formula (4) from $E_{y}(x, \omega)$ and $B_{x}(x, \omega)$ at the Earth's surface. Besides $b$ and $\omega, Z_{\mathrm{w}}(b, \omega)$ is affected by the Earth's conductivity structure, and as mentioned in Section 2.1, it can be calculated recursively for a layered Earth.

\subsection{Convolution integrals in the space domain}

Similarly to the derivation of Eq. (6) from formula (2), Eq. (9) leads to the convolution integral

$$
\begin{aligned}
E_{y}(x) & =-\frac{1}{\mu_{0} \sqrt{2 \pi}} \int_{-\infty}^{\infty} Z_{\mathrm{w}}\left(x-x^{\prime}\right) B_{x}\left(x^{\prime}\right) d x^{\prime} \\
& =-\frac{1}{\mu_{0} \sqrt{2 \pi}} \int_{-\infty}^{\infty} Z_{\mathrm{w}}\left(x^{\prime}\right) B_{x}\left(x-x^{\prime}\right) d x^{\prime}
\end{aligned}
$$

where $E_{y}(x), B_{x}(x)$ and $Z_{\mathrm{w}}(x)$ are the inverse Fourier transforms of $E_{y}(b), B_{x}(b)$ and $Z_{\mathrm{w}}(b)$ (Eq. (5)). The $\omega$ dependence is not written explicitly. Note the difference in the use of the $2 \pi$ factor compared to the formulas presented, for example, by Pirjola et al. (2004). This is due to different conventions in the Fourier transform pair.

Equation (10) shows that $E_{y}$ at a given location $x$ depends on the values of $B_{x}$ at other locations $x^{\prime}$, and the dependence is determined by the (kernel) function $Z_{\mathrm{w}}\left(x-x^{\prime}\right)$. If $B_{x}$ data are available from a chain of magnetometer stations with different $x$ coordinates Eq. (10) provides a simple means to calculate the electric field to be applied, for example, to computing GIC in a technological network. It is very important to note that $Z_{\mathrm{w}}(x)$ has nothing to do with the surface impedance function $Z_{\mathrm{s}}(x)$ defined by Eq. (8) (the $\omega$ 
dependence is implicit in both functions). Mathematically this is due to the fact that the (inverse) Fourier transform of a ratio (Eq. (9)) is not equal to the ratio (Eq. (8)) of the (inverse) Fourier transforms of the top and bottom separately. Even the units of $Z_{\mathrm{w}}(x)\left[\Omega \mathrm{m}^{-1}\right]$ and of $Z_{\mathrm{s}}(x)[\Omega]$ are different.

If $Z_{\mathrm{w}}(b)$ is (or can be assumed to be) independent of $b$ and equal to $Z_{0}$ Eq. (5) gives that $Z_{\mathrm{w}}(x)$ equals $Z_{0} \sqrt{2 \pi} \delta(x)$ where $\delta(x)$ is the Dirac delta function. Substituting this into Eq. (10) shows that $E_{y}(x)$ only depends on $B_{x}(x)$ at the same location $x$, which is a result also obtainable by using Eqs. (5) and (9). This resembles the plane wave situation with $Z_{0}$ corresponding to the plane wave surface impedance $Z_{\mathrm{p}}$ (Section 2.2). In the strict plane wave case, $E_{y}$ and $B_{x}$ should be independent of $x$. Thus, the fact that the dependence on $x$ need not vanish from Eq. (10) even in the present delta function case provides a hint that it is sometimes possible to use the plane wave relation between $E_{y}$ and $B_{x}$ locally (see Pirjola et al., 2004; Viljanen et al., 2004). As indicated by Pirjola et al. (2004), who consider a uniform Earth (cf. Section 2.6), $Z_{\mathrm{w}}(x)$ seems to approach the delta function when the period decreases and/or the Earth's conductivity increases. This is in agreement with the result that source effects distorting the plane wave assumption and referred to in Section 2.2 are more serious for long periods and resistive Earth structures, both of which increase the skin depth (see numerical values mentioned in Section 2.2).

Another extreme theoretical case is obtained by assuming that $Z_{\mathrm{w}}(b)$ is proportional to the delta function $\delta(b)$. Equation (5) then shows that $Z_{\mathrm{w}}(x)$ is independent of $x$, which by formula (10) also makes $E_{y}$ independent of $x$ and proportional to the integral of $B_{x}(x)$ over all values of $x$. By formula (4), this integral gives the Fourier transform of $B_{x}(x)$ at $b=0$. In other words, $E_{y}$ only gets a constant plane wave contribution $(b=0)$ at every value of $x$. This can actually be directly seen from the assumption of $Z_{\mathrm{w}}(b)$ to be proportional to $\delta(b)$, which is zero for $b \neq 0$.

\subsection{Uniform Earth}

Let us consider a uniform Earth characterised by the permeability $\mu$, permittivity $\varepsilon$ and conductivity $\sigma$. The Earth's permeability and permittivity are commonly in geoelectromagnetic studies, as well as in the numerical calculations presented in Section 3, assumed to equal $\mu_{0}$ and $5 \varepsilon_{0} \quad\left(\varepsilon_{0}\right.$ is the vacuum (and air) permittivity $=$ $8.854 \cdot 10^{-12} \mathrm{~A} \mathrm{~s} \mathrm{~V}^{-1} \mathrm{~m}^{-1}$ ), respectively, although the latter does not have any effect in practice in the range of periods considered. However, in this section $\mu$ and $\varepsilon$ need not be specified, and so they are arbitrary.

Making first the plane wave assumption and considering a single angular frequency $\omega$, i.e. fields Fourier transformed from the time to the frequency domain, we easily obtain

$$
Z_{\mathrm{p}}(\omega)=\frac{\omega \mu}{k}
$$

where the propagation constant $k$ is given by

$$
k=\sqrt{\omega^{2} \mu \varepsilon-i \omega \mu \sigma}
$$

(e.g. Pirjola, 1982; Pirjola et al., 2004). An application of the expression (11) makes the convolution integral (7) read

$$
E_{y}(t)=-\frac{1}{\mu_{0}} \sqrt{\frac{\mu}{\varepsilon}} \int_{0}^{\infty} g(t-u) e^{-\frac{\sigma u}{2 \varepsilon}} J_{0}\left(i \frac{\sigma u}{2 \varepsilon}\right) d u
$$

The derivation of Eq. (13) is based on the determination of the function $C_{\mathrm{p}}(t)$ included in Eq. (7) by inverse Fourier transforming the function $Z_{\mathrm{p}}(\omega) / i \omega$, which in this case equals $\mu / i k(\omega)$. As presented by Pirjola (1982, p. 23), the inverse Fourier transform can be calculated by utilising an integral expression of the Bessel function of the zero order $J_{0}$. A comparison of Eq. (13) with the corresponding formula by Pirjola (1982, p. 23) indicates a small difference, which results from the neglect of the assumption that $\mu$ would equal $\mu_{0}$ now.

In practical geoelectromagnetic situations $\varepsilon$ is around $5 \cdot 10^{-11} \mathrm{~A} \mathrm{~s} \mathrm{~V}^{-1} \mathrm{~m}^{-1}$ and $\sigma$ is in the order of $10^{-5} \Omega^{-1} \mathrm{~m}^{-1}$ or larger. Thus the characteristic time $\varepsilon / \sigma$ is about $5 \mu$ s or smaller, and so relevant times in geoelectromagnetics being in the order of a second at the minimum are very much longer than $\varepsilon / \sigma$. This indicates that the absolute value of the argument of the Bessel function in Eq. (13) is very large except for an insignificant time interval near $u=0$. Consequently, we replace the Bessel function term $J_{0}(i \sigma u / 2 \varepsilon)$ by the approximate expression $e^{\sigma u / 2 \varepsilon} \sqrt{\varepsilon / \pi \sigma u}$ valid when the absolute value of the argument is large. Equation (13) then reduces to

$$
E_{y}(t)=-\sqrt{\frac{\mu}{\mu_{0}}} \frac{1}{\sqrt{\pi \mu_{0} \sigma}} \int_{0}^{\infty} \frac{g(t-u)}{\sqrt{u}} d u
$$

An alternative, but completely equivalent way, to derive Eq. (14) is to note that in practical geoelectromagnetic situations $\sigma \gg \omega \varepsilon$, which enables the neglect of the first term in the square root in formula (12). Calculating $C_{\mathrm{p}}(t)$ by inverse Fourier transforming $\mu / i k(\omega)=\mu / i \sqrt{-i \omega \mu \sigma}$ directly leads to Eq. (14) then. Both formula (13) and formula (14) confirm the statement about the causality mentioned in Section 2.2 since $E_{y}(t)$ only depends on values of $g$ at times earlier than $t$. Equations (13) and (14) can, of course, also be written following the first integral of formula (7). The integration limits would then be $-\infty$ and $t$. In practice, Eq. (14), usually with the assumption that $\mu$ and $\mu_{0}$ are equal, is applicable to estimating the electric field from magnetic data in the time domain. This formula is also included in the fundamental paper of magnetotellurics by Cagniard (1953). By considering a fictitious step-like change of $B_{x}$, Pirjola (1982, pp. 24-25) explicitly demonstrates that the $E_{y}$ values obtained from Eqs. (13) and (14) are practically the same although, strictly speaking, $E_{y}[(14)]$ becomes infinite as " $1 / \sqrt{0}$ " at the time of the step whereas $E_{y}[(13)]$ remains finite.

From the mathematical point of view, it is still necessary to emphasise that the inverse Fourier transform (5) requires the knowledge of the function $F(\omega)$ for all values of $\omega$ from $-\infty$ to $+\infty$. In the present uniform Earth case, the function transformed above includes a square root of a complex variable (either exactly $\sqrt{\omega^{2} \mu \varepsilon-i \omega \mu \sigma}$ or approximately $\sqrt{-i \omega \mu \sigma}$ ). Therefore, as explained by Pirjola (1982, p. 22), special care is needed to define the correct argument ranges in the complex plane in which the square root lies for positive and negative values of $\omega$. 
Equation (14) shows explicitly that $E_{y}(t)$ is not proportional to the time derivative $g(t)$ of $B_{x}(t)$ but is also affected by past values of $g(t)$. However, the square root term $\sqrt{u}$ at the bottom of the integral implies that the weight of past values decreases with an increasing time difference. Equation (14) also indicates that $E_{y}(t)$ is not proportional to $B_{x}(t)$ either. (Note that these conclusions about the relations of $E_{y}(t)$ to $g(t)$ and to $B_{x}(t)$ are not due to the use of the approximate equation (14) instead of the exact formula (13).) Referring to Section 2.2, Eq. (11), with the definition (12), confirms the conclusions by showing that $Z_{\mathrm{p}}(\omega)$ is neither independent of $\omega$ nor linearly dependent on $\omega$. Replacing $k$ by $\sqrt{-i \omega \mu \sigma}$ in formula (11) shows that $Z_{\mathrm{p}}(\omega)$ is proportional to the square root of $\omega$, which means that a uniform Earth is a high-pass filter from the magnetic to the electric field. Substituting $Z_{\mathrm{p}}(\omega)$ from Eq. (11) with $k=\sqrt{-i \omega \mu \sigma}$ into formula (2) shows that, in the case of a uniform Earth, the phase of $-E_{y}$ is always 45 degrees ahead of that of $B_{x}$. (This is directly seen for $\omega>0$ but requires more examination when $\omega<0$.)

We now neglect the plane wave assumption and consider the $E$ polarisation case discussed in Sections 2.3, 2.4 and 2.5 but the Earth is kept uniform. The single angular frequency $\omega$ considered is an implicit argument. It can be shown that $Z_{\mathrm{w}}(b)$ has the expression

$$
Z_{\mathrm{w}}(b)=\frac{i \omega \mu}{\sqrt{b^{2}-k^{2}}}
$$

(Pirjola, 1982, p. 51). Taking the inverse Fourier transform (5) of $Z_{\mathrm{w}}(b)$ gives $Z_{\mathrm{w}}(x)$ :

$$
Z_{\mathrm{w}}(x)=\sqrt{\frac{\pi}{2}} \omega \mu H_{0}^{(2)}(k x)
$$

where an integral formula for the Hankel function of the second kind and of the zero order $H_{0}^{(2)}$ has been used (Pirjola, 1982, p. 128). Comparing Eq. (16) to the corresponding expression presented by Pirjola et al. (2004), a small difference caused by a different use of the $2 \pi$ factor in the Fourier transform pair is observed (cf. Section 2.5).

Let us consider the dependence of $Z_{\mathrm{w}}(b)$ given by Eq. (15) on the wavenumber $b$. Since $Z_{\mathrm{w}}(b)$ is an even function of $b$ we may restrict to non-negative $b$ values. Equation (15) directly shows that $Z_{\mathrm{w}}(b)$ goes to zero when $b$ approaches infinity. The maximum value of $\left|Z_{\mathrm{w}}(b)\right|$ is obtained at the value of $b$ that makes the real part of the function inside the square root at the bottom of Eq. (15) (= $\operatorname{Re}\left(b^{2}-k^{2}\right)$ ) equal to zero, i.e. when $b=\omega \sqrt{\mu \varepsilon}$ (implicitly assuming that $\omega$ is non-negative). At $b$ values smaller than $\omega \sqrt{\mu \varepsilon},\left|Z_{\mathrm{w}}(b)\right|$ is an increasing function of $b$, and at larger values it decreases with $b$. As mentioned above, $\sigma \gg \omega \varepsilon$ in practical geoelectromagnetic situations, making $\left|\operatorname{Im}\left(k^{2}\right)\right| \gg \operatorname{Re}\left(k^{2}\right)$. Thus, the term $b^{2}-k^{2}$ is completely dominated by $\operatorname{Im}\left(k^{2}\right)$ when $b$ is small, and so the $b$ dependence of $Z_{\mathrm{w}}(b)$ at the smallest values of $b$, including the maximum of $\left|Z_{\mathrm{w}}(b)\right|$, cannot be seen in practice. This means that $\left|Z_{\mathrm{w}}(b)\right|$ looks practically like having its maximum at $b=0$ and decreasing with $b$.

Pirjola et al. (1999) indicate that in the case of a layered
Earth $Z_{\mathrm{w}}(b)$ can be approximated by

$$
Z_{\mathrm{w}}(b)=\frac{i \omega \mu_{0}}{\sqrt{b^{2}-k_{\mathrm{u}}^{2}}}
$$

The "equivalent propagation constant" $k_{\mathrm{u}}$, which is independent of $b$, is defined by

$$
k_{\mathrm{u}}=k_{\mathrm{u}}(\omega)=\frac{\omega \mu_{0}}{Z_{\mathrm{p}}(\omega)}
$$

where $Z_{\mathrm{p}}(\omega)$ is the plane wave $(b=0)$ surface impedance (Section 2.2) for the particular layered Earth structure. Similarly to Eqs. (15) and (16), formula (17) gives $Z_{\mathrm{w}}(x)$ the approximate expression

$$
Z_{\mathrm{w}}(x)=\sqrt{\frac{\pi}{2}} \omega \mu_{0} H_{0}^{(2)}\left(k_{\mathrm{u}} x\right)
$$

\section{Numerical Examples}

We now present numerical results associated with the equations discussed in Section 2. Following the statement in Section 2.6, the permeability and permittivity of the Earth are set equal to $\mu_{0}$ and $5 \varepsilon_{0}$, respectively.

Figure 1 presents the absolute value of the plane wave surface impedance $\left|Z_{\mathrm{p}}(\omega)\right|$ as a function of the frequency ( $f=\omega / 2 \pi=1 / T, T=$ period) for a two-layer Earth in which the thickness of the upper layer and the conductivity of the lower layer are $150 \mathrm{~km}$ and $2.6 \Omega^{-1} \mathrm{~m}^{-1}$, respectively. The three curves from the bottom to the top, two of which are practically straight lines in this loglog plot, are associated with the upper layer conductivities $1000 \Omega^{-1} \mathrm{~m}^{-1}$ (blue), $0.01 \Omega^{-1} \mathrm{~m}^{-1}$ (green) and $10^{-5} \Omega^{-1} \mathrm{~m}^{-1}$ (red). In each case, $\left|Z_{\mathrm{p}}(\omega)\right|$ varies much with the frequency indicating no proportionality between $E_{y}(t)$ and $B_{x}(t)$ but the larger the Earth's conductivity the smaller the relative change of $\left|Z_{\mathrm{p}}(\omega)\right|$ in the frequency range considered. Since $\left|Z_{\mathrm{p}}(\omega)\right|$ grows with the frequency the Earth behaves as a high-pass filter. The curves in Fig. 1 also imply the well-known fact that a lower conductivity and a higher frequency result in larger horizontal electric fields (assuming the same magnetic field). The former conclusion emphasises the risk for large GIC in networks lying in resistive areas. Note that an Earth conductivity of $1000 \Omega^{-1} \mathrm{~m}^{-1}$ is too large to be encountered in practical situations.

The Earth model included in Fig. 2, which presents $\left|Z_{\mathrm{p}}(\omega)\right|$ as a function of the frequency, should be considered less probable in practice due to the poorly-conducting halfspace $\left(10^{-5} \Omega^{-1} \mathrm{~m}^{-1}\right)$ below a thin surface layer $(10 \mathrm{~km})$ but it offers an interesting conclusion about $E_{y}(t)$ and $B_{x}(t)$ in one case. The three curves from the bottom to the top correspond to the upper layer conductivities $1000 \Omega^{-1} \mathrm{~m}^{-1}$ (blue), $0.01 \Omega^{-1} \mathrm{~m}^{-1}$ (green) and $1.1 \cdot 10^{-5} \Omega^{-1} \mathrm{~m}^{-1}$ (red). The skin depth for the largest conductivity is much less than the thickness of the upper layer even at the lowest frequency considered, so the lower layer does not play any role for the blue curve, which further means that the blue curves of Figs. 1 and 2 are identical (note the different frequency ranges considered). The variation of $\left|Z_{\mathrm{p}}(\omega)\right|$ with frequency is quite small in the green curve indicating a 


\section{$\left|Z_{p}\right|[\Omega]$}

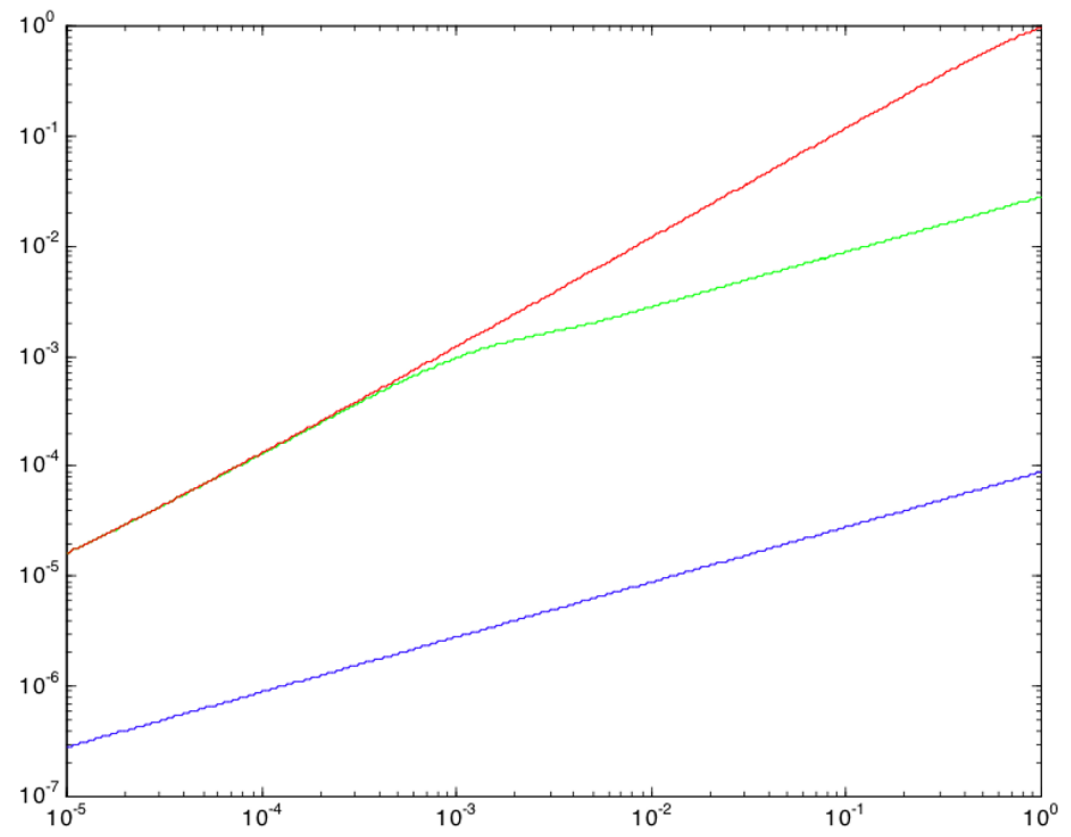

\section{$f=\omega / 2 \pi[\mathrm{Hz}]$}

Fig. 1. Absolute value of the plane wave surface impedance $Z_{\mathrm{p}}(\omega)$ as a function of the frequency $f=\omega / 2 \pi$. The Earth has two layers. The thickness of the upper layer and the conductivity of the lower layer are $150 \mathrm{~km}$ and $2.6 \Omega^{-1} \mathrm{~m}^{-1}$, respectively. The three curves from the bottom to the top refer to the upper layer conductivities $1000 \Omega^{-1} \mathrm{~m}^{-1}$ (blue), $0.01 \Omega^{-1} \mathrm{~m}^{-1}$ (green) and $10^{-5} \Omega^{-1} \mathrm{~m}^{-1}$ (red).

\section{$\left|Z_{p}\right|[\Omega]$}

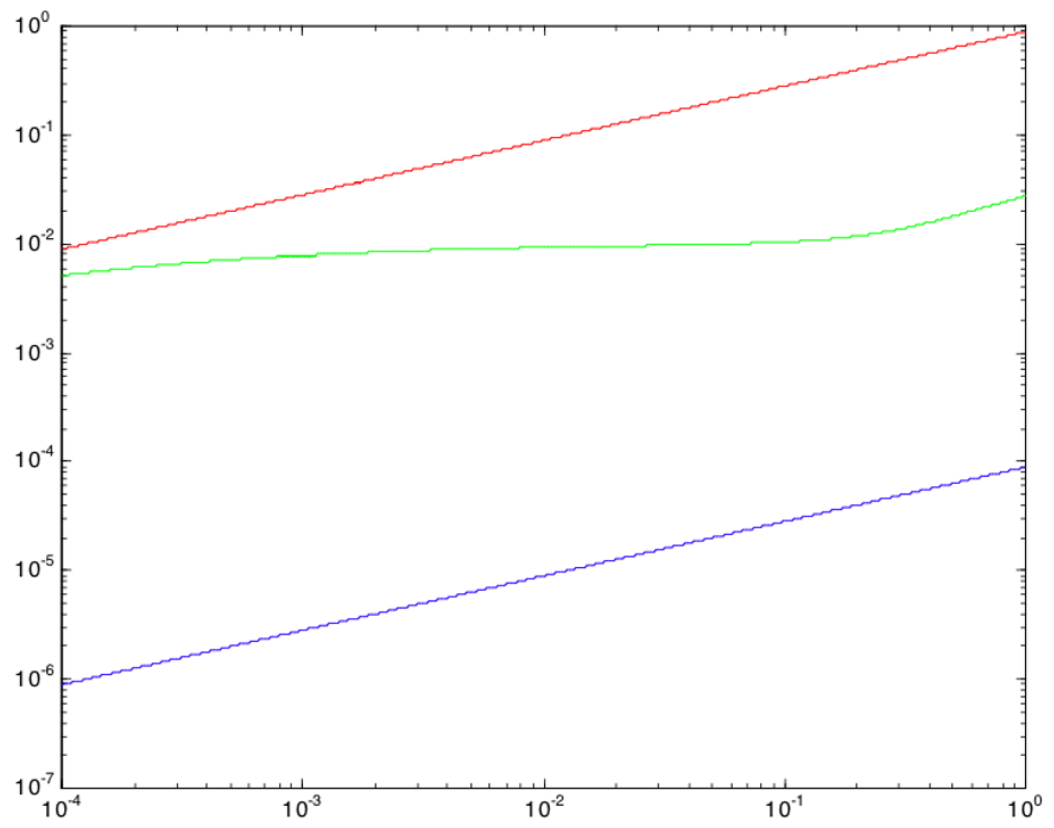

$$
f=\omega / 2 \pi[\mathrm{Hz}]
$$

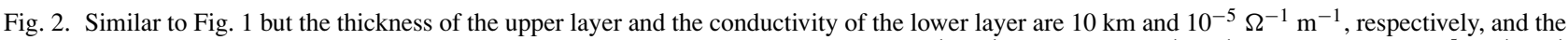
three curves from the bottom to the top refer to the upper layer conductivities $1000 \Omega^{-1} \mathrm{~m}^{-1}$ (blue), $0.01 \Omega^{-1} \mathrm{~m}^{-1}$ (green) and $1.1 \cdot 10^{-5} \Omega^{-1} \mathrm{~m}^{-1}$ (red). 


\section{$\left|Z_{p}\right|[\Omega]$}

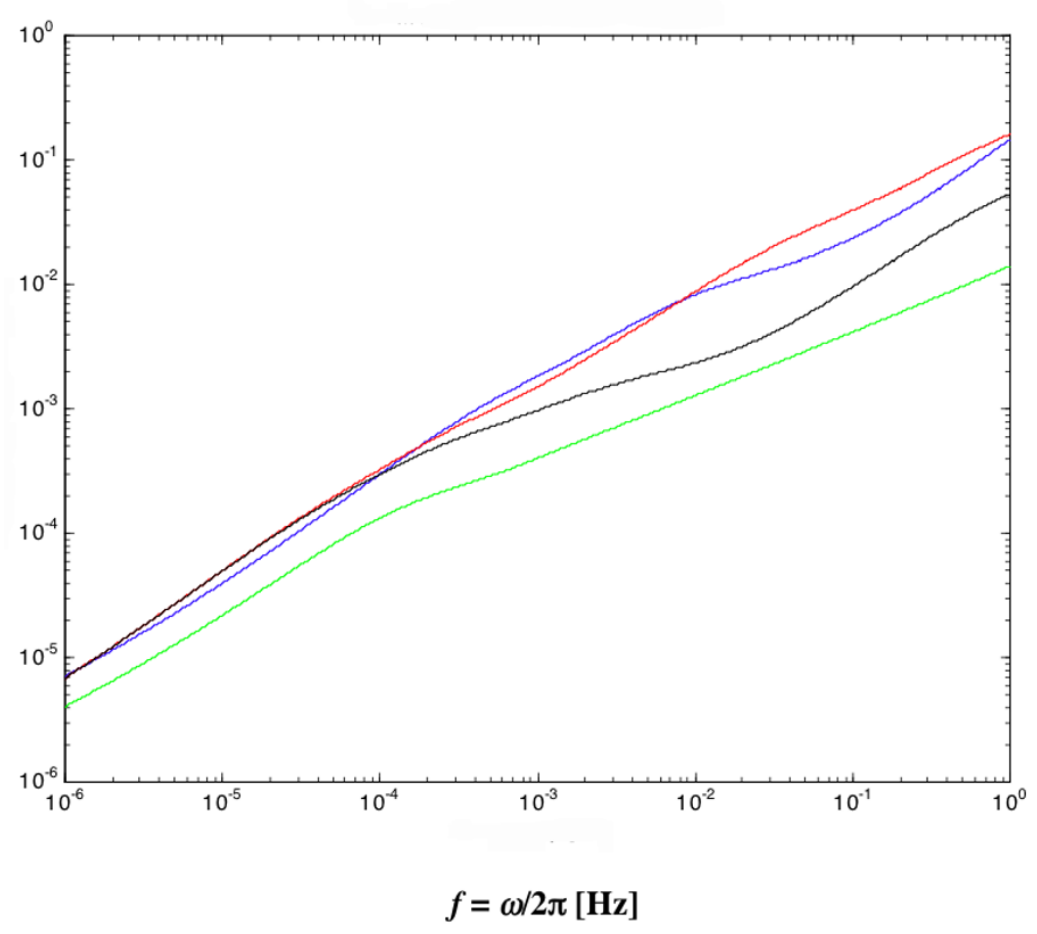

Fig. 3. Absolute value of the plane wave surface impedance $Z_{\mathrm{p}}(\omega)$ as a function of the frequency $f=\omega / 2 \pi$. Four different Canadian layered Earth models are considered: "Quebec", 5 layers, blue (Boteler and Pirjola, 1998): thicknesses $=[15,10,125,200, \infty] \mathrm{km}$, resistivities $=[20000,200$, $1000,100,3] \Omega \mathrm{m}$. "Ontario-Model 1", 3 layers, green: thicknesses $=[0.3,200, \infty] \mathrm{km}$, resistivities $=[100,20,1] \Omega \mathrm{m}$. "Southern Manitoba", 8 layers, red: thicknesses $=[0.02,0.18,14.8,10,75,300,200, \infty] \mathrm{km}$, resistivities $=[20,100,40000,2000,1000,100,10,1] \Omega \mathrm{m} . "$ British Columbia", 8 layers, black (Boteler and Pirjola, 1998): thicknesses $=[4,6,5,20,65,300,200, \infty] \mathrm{km}$, resistivities $=[500,150,20,100,300,100$, $10,1] \Omega \mathrm{m}$.

rough proportionality between $E_{y}(t)$ and $B_{x}(t)$. Figure 2 only shows the absolute value of the surface impedance, and definite conclusions about the dependence of the complex quantity $Z_{\mathrm{p}}(\omega)$ on $\omega$ would require the investigation of its phase angle, too, but in this paper we are mostly interested in the magnitudes of the geoelectric field (via the absolute values of the surface impedance), and so the phases are less important.

Figures 3 and 4 , which present $\left|Z_{\mathrm{p}}(\omega)\right|$ and $\left|Z_{\mathrm{p}}(\omega) / i \omega\right|$, refer to four realistic layered Earth models from Canada:

- "Quebec", 5 layers, blue (Boteler and Pirjola, 1998) layer thicknesses $=[15,10,125,200, \infty] \mathrm{km}$ layer resistivities $=[20000,200,1000,100,3] \Omega \mathrm{m}$

- "Ontario-Model 1", 3 layers, green layer thicknesses $=[0.3,200, \infty] \mathrm{km}$ layer resistivities $=[100,20,1] \Omega \mathrm{m}$

- "Southern Manitoba", 8 layers, red layer thicknesses $=[0.02,0.18,14.8,10,75,300,200$, $\infty] \mathrm{km}$ layer resistivities $=[20,100,40000,2000,1000,100$, $10,1] \Omega \mathrm{m}$

- "British Columbia", 8 layers, black (Boteler and Pirjola, 1998)

layer thicknesses $=[4,6,5,20,65,300,200, \infty] \mathrm{km}$ layer resistivities $=[500,150,20,100,300,100,10$, 1] $\Omega \mathrm{m}$

Similarly to Fig. 1, a clear high-pass filter property is exhib- ited by every model in Fig. 3 whereas the curves in Fig. 4 have a low-pass filter character. A comparison between Figs. 3 and 4 shows that $\left|Z_{\mathrm{p}}(\omega) / i \omega\right|$ changes relatively less than $\left|Z_{\mathrm{p}}(\omega)\right|$ with $\omega$ indicating that $E_{y}(t)$ resembles more $g(t)$ than $B_{x}(t)$. This confirms the use of the magnetic field time derivative as a proxy for the geoelectric field and GIC.

Figure 5 depicts $\left|Z_{\mathrm{s}}(x, \omega)\right|$ (Eq. (8)) as a function of $x$ when the electric and magnetic fields are produced by a line current parallel to the $y$ axis at $x=0$ and at the height $-z=110 \mathrm{~km}$, and the period is $T=300 \mathrm{~s}(=2 \pi / \omega)$. The Earth is assumed to have the five-layer "Quebec" structure. The computation of the fields was performed by applying the Fast Hankel Transform (Johansen and Sørensen, 1979; see also Pirjola, 1985; Pirjola and Boteler, 2002). The limit that $\left|Z_{\mathrm{s}}(x, \omega)\right|$ approaches at large values of $x$ equals the absolute value of the plane wave surface impedance corresponding to the situation with no $x$ variation. We can also easily verify that the real and imaginary parts of $Z_{\mathrm{s}}(x, \omega)$ approach the corresponding plane wave quantities separately. This behaviour of $Z_{\mathrm{s}}(x, \omega)$ is in agreement with the statement about source effects in Section 2.2.

Figure 5 shows that $\left|Z_{\mathrm{s}}(x, \omega)\right|$ varies much with $x$ and that the statement given by Albertson and Van Baelen (1970) that the plane wave case would result in the largest electric field if the magnetic field is kept constant is not true. Anyway, it can be seen from Fig. 5 that at and near $x=0$ the plane wave electric field is larger than that produced by a line current (for the same magnetic field), and in fact, this 


\section{$\left|Z_{p} / \mathbf{i} \omega\right|[\Omega \mathrm{s}]$}

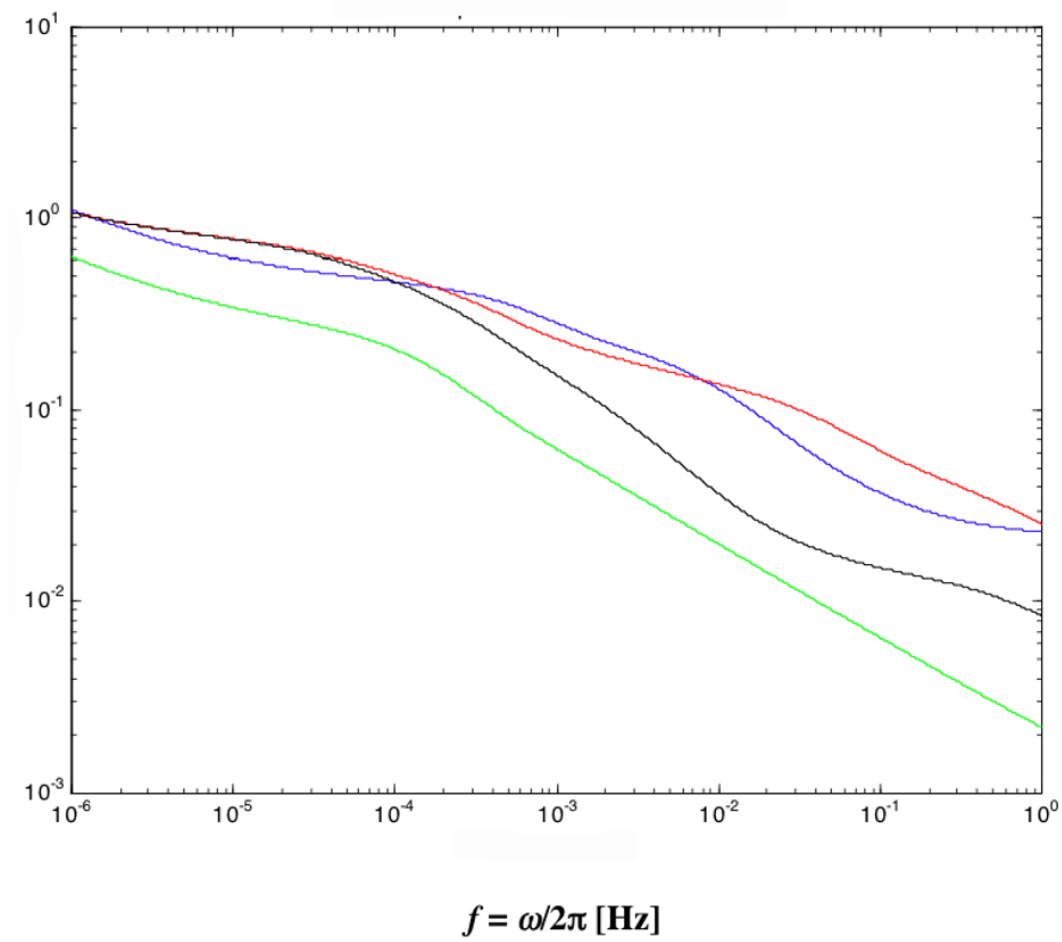

Fig. 4. Absolute value of the quantity $Z_{\mathrm{p}}(\omega) / i \omega$ as a function of the frequency $f=\omega / 2 \pi$ where $Z_{\mathrm{p}}(\omega)$ is the plane wave surface impedance and $\omega$ is the angular frequency. Thus, the curves represent the transfer function between the electric field and the time derivative of the magnetic field. Similarly to Fig. 3, four different Canadian layered Earth models are considered.

\section{$\left|Z_{s}\right|[\Omega]$}

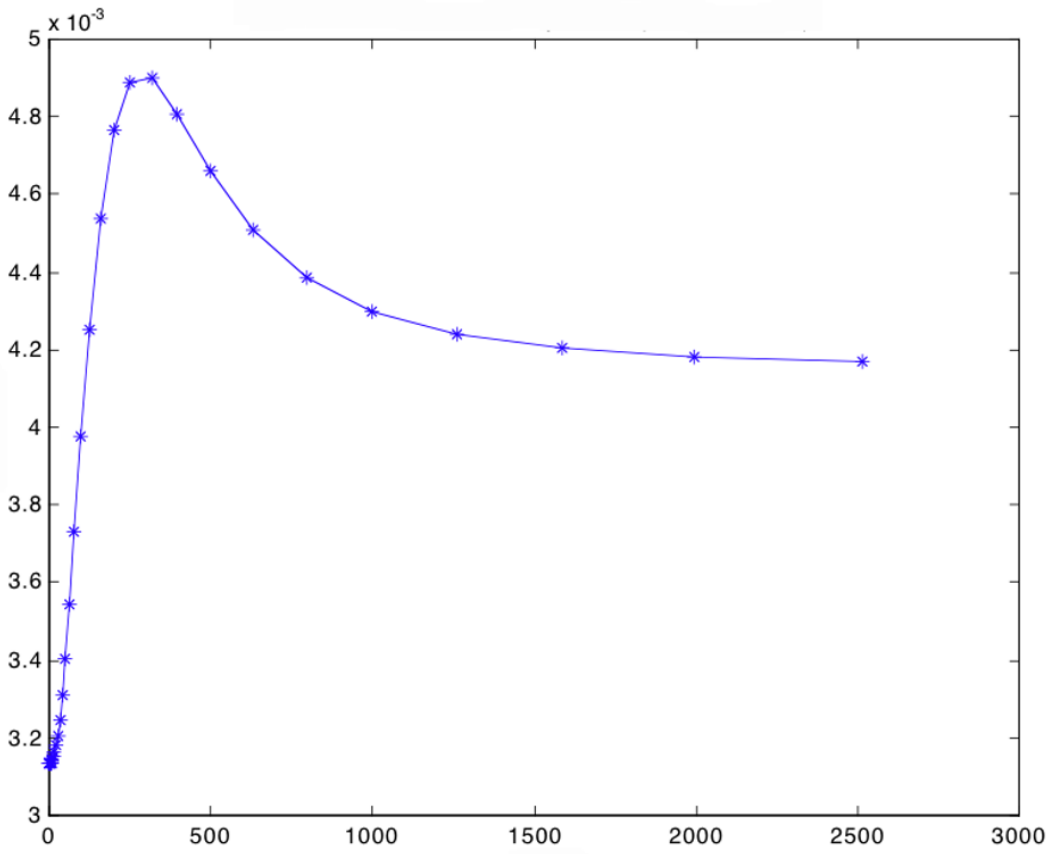

$x[\mathrm{~km}]$

Fig. 5. Absolute value of the space-domain surface impedance $Z_{\mathrm{s}}(x, \omega)$ (Eq. (8)) as a function of the $x$ coordinate. The electric and magnetic fields are produced by an infinitely long line current parallel to the $y$ axis at $x=0$ and at the height of $-z=110 \mathrm{~km}$. The period considered is $T=300 \mathrm{~s}$ $(=2 \pi / \omega)$. The Earth has the five-layer "Quebec" structure (see the caption of Fig. 3). 


\section{$\left|Z_{w}\right|, \operatorname{Re}\left(Z_{w}\right), \operatorname{Im}\left(Z_{w}\right)[\Omega]$}

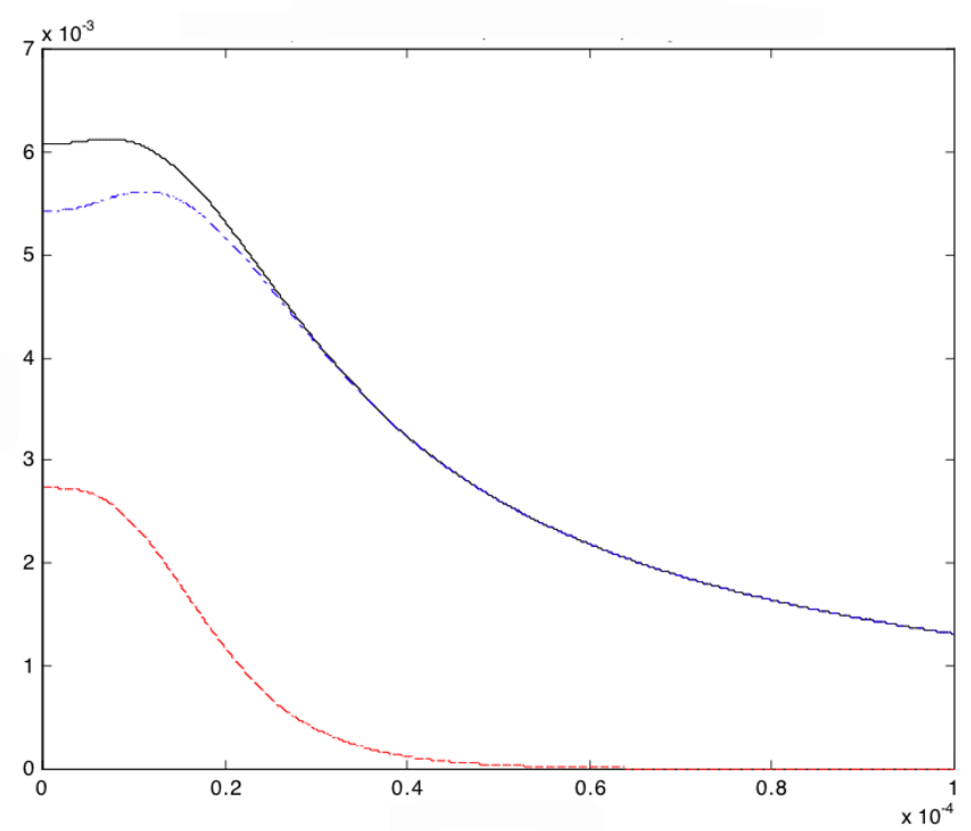

$b\left[\mathrm{~m}^{-1}\right]$

Fig. 6. Absolute value (black solid curve) and real (red dashed curve) and imaginary (blue dot-dashed curve) of the surface impedance $Z_{\mathrm{w}}(b, \omega)$ (Eq. (9)) as a function of the wavenumber $b$. The period considered is $T=60 \mathrm{~s}(=2 \pi / \omega)$. The Earth has a layered structure: "Central Finland", 5 layers (Viljanen and Pirjola, 1994): thicknesses $=[12,22,16,50, \infty] \mathrm{km}$, resistivities $=[30000,3000,50,1000,5000] \Omega \mathrm{m}$.

\section{$\left|Z_{w}\right|, \operatorname{Re}\left(Z_{w}\right), \operatorname{Im}\left(Z_{w}\right)[\Omega]$}

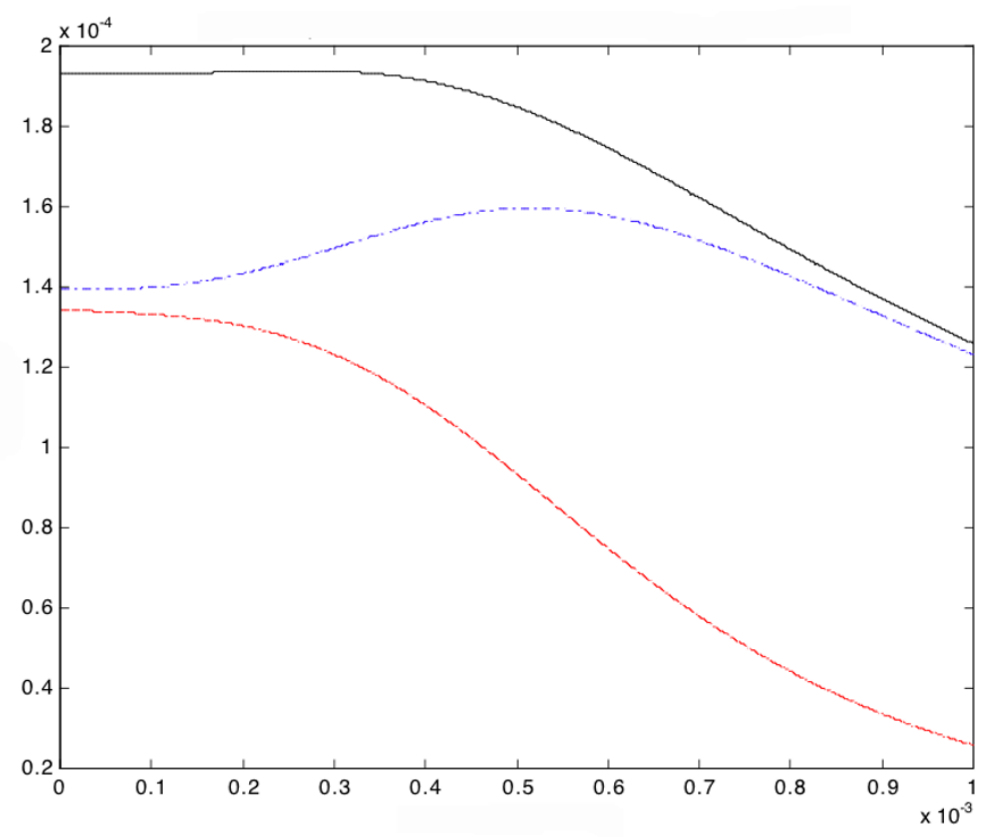

$b\left[\mathbf{m}^{-1}\right]$

Fig. 7. Similar to Fig. 6 but the Earth has a different layered structure: "Nova Scotia", 3 layers: thicknesses $=[4,196, \infty]$ km, resistivities $=[0.3,10$, $0.3] \Omega \mathrm{m}$.

is the location that Albertson and Van Baelen only consider. Häkkinen et al. (1989) use a more sophisticated ionospheric electrojet current system model and demonstrate even more pronounced and unpredictable differences from the plane wave case at particular values of $x$ when investigating the magnetotelluric apparent resistivity $\rho_{\mathrm{a}}$ as a function of the period. The quantity $\rho_{\mathrm{a}}$ is proportional to the square of the absolute value of the surface impedance times the period, so 


\section{$\left|Z_{w}\right|, \operatorname{Re}\left(Z_{w}\right), \operatorname{Im}\left(Z_{w}\right)[\Omega]$}

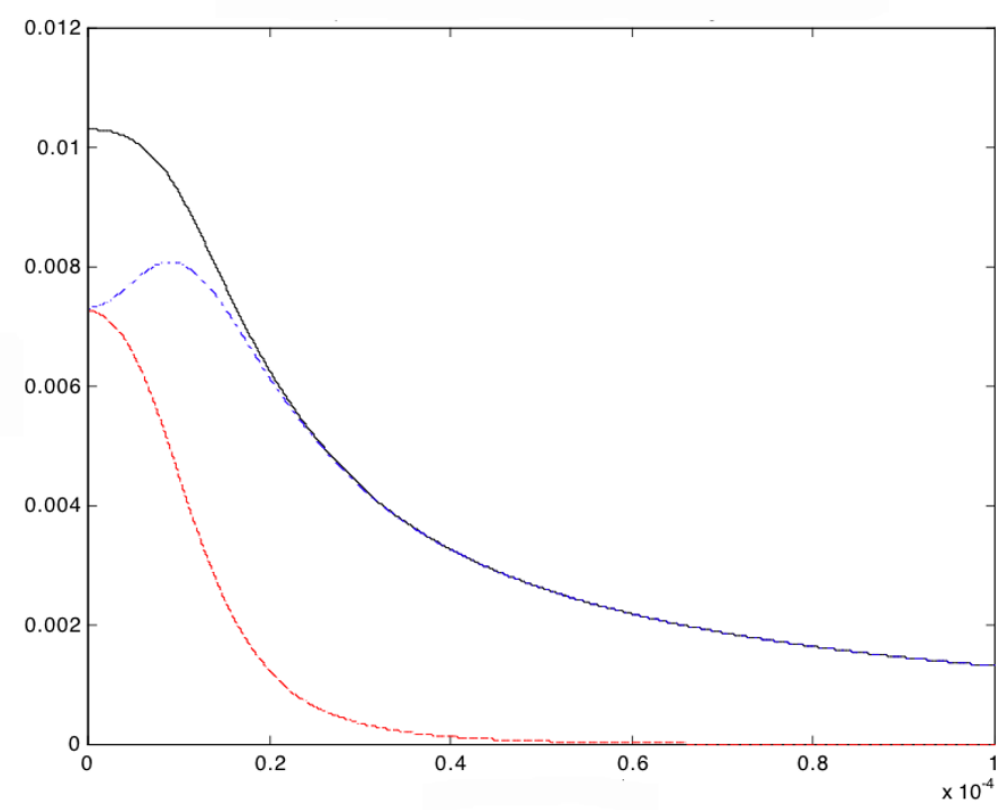

$b\left[\mathrm{~m}^{-1}\right]$

Fig. 8. Similar to Fig. 6 but the Earth is assumed to have the five-layer "Quebec" structure (see the caption of Fig. 3).

\section{$\left|Z_{w}\right|, \operatorname{Re}\left(Z_{w}\right), \operatorname{Im}\left(Z_{w}\right)[\Omega]$}

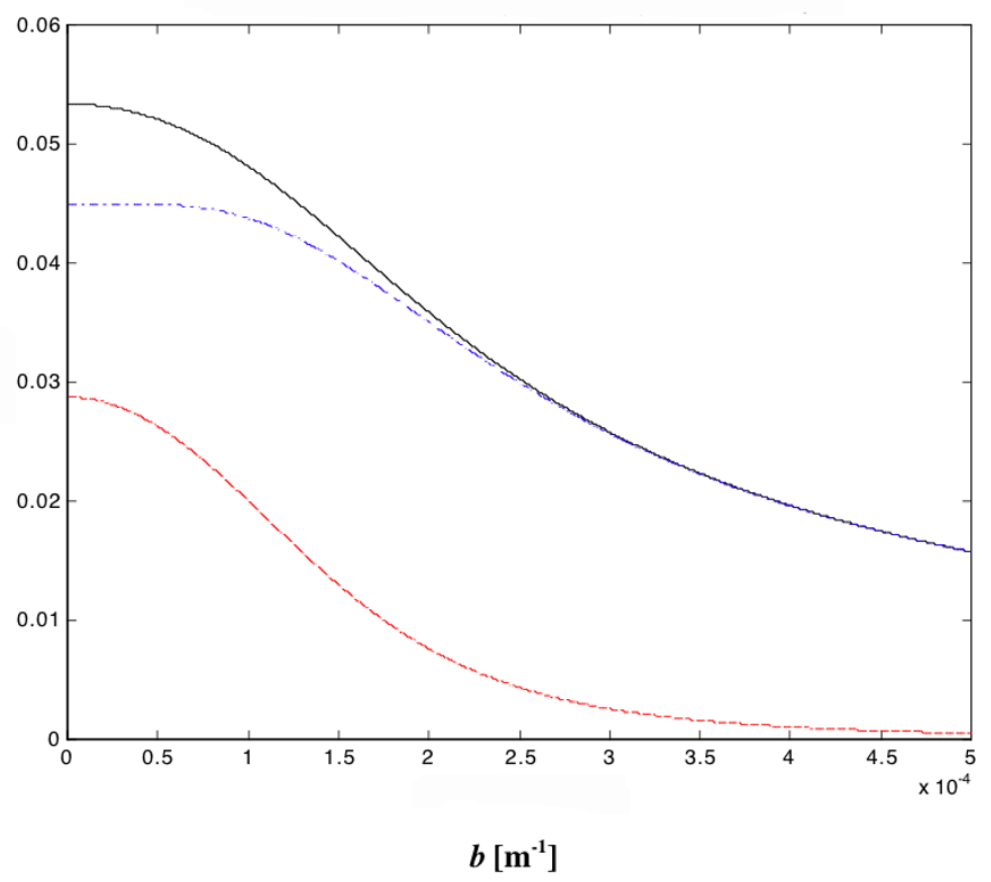

Fig. 9. Similar to Fig. 6 but the period is $1 \mathrm{~s}$ and the Earth is assumed to have the eight-layer "British Columbia" structure (see the caption of Fig. 3).

the behaviour of $\rho_{\mathrm{a}}$ also reflects the properties of the surface impedance.

We now compute $Z_{\mathrm{w}}(b)$ (Eq. (9), $\omega$ is implicit) for specified realistic layered Earth models. Figures 6-11 then depict the real and imaginary parts and the absolute value of $Z_{\mathrm{w}}(b)$ as functions of $b$. Note the differences in the scales both on the horizontal and on the vertical axes. Figures 6,7, 8 and 10 refer to a period $T=60 \mathrm{~s}(=2 \pi / \omega)$ and to Earth models corresponding "Quebec" and "British Columbia" introduced above and to "Central Finland" and "Nova Scotia" (Canada): 
$I Z_{w} \mid, \operatorname{Re}\left(Z_{w}\right), \operatorname{Im}\left(Z_{w}\right)[\Omega]$

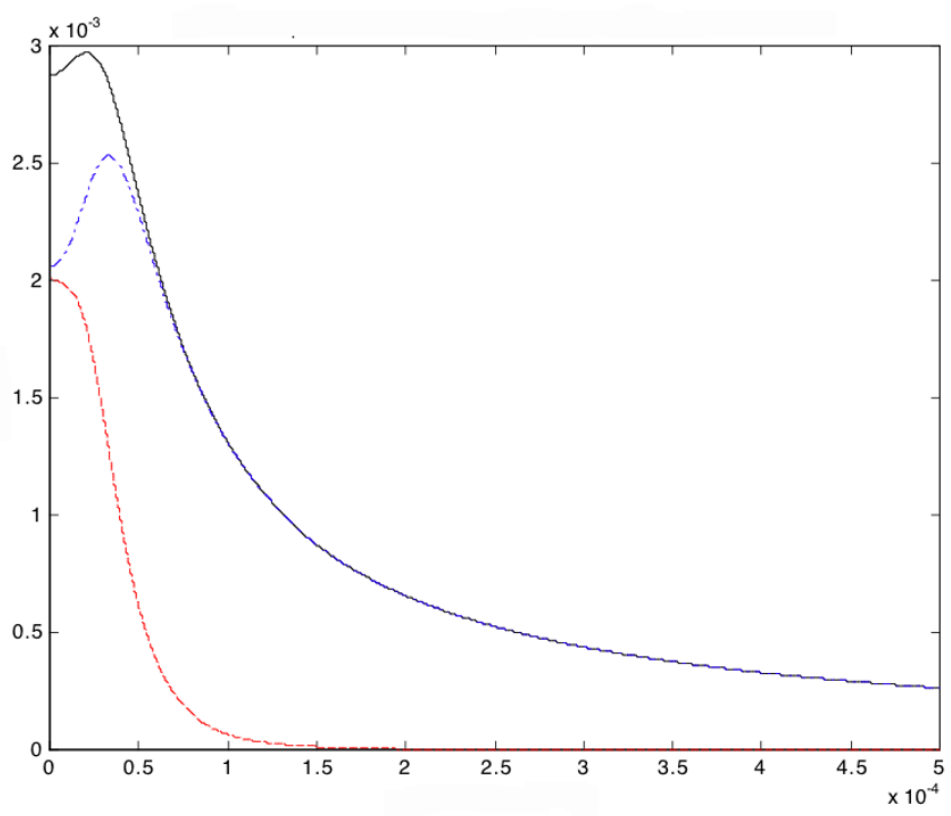

$b\left[\mathrm{~m}^{-1}\right]$

Fig. 10. Similar to Fig. 9 but the period is $60 \mathrm{~s}$.

$\left|Z_{w}\right|, \operatorname{Re}\left(Z_{w}\right), \operatorname{Im}\left(Z_{w}\right)[\Omega]$

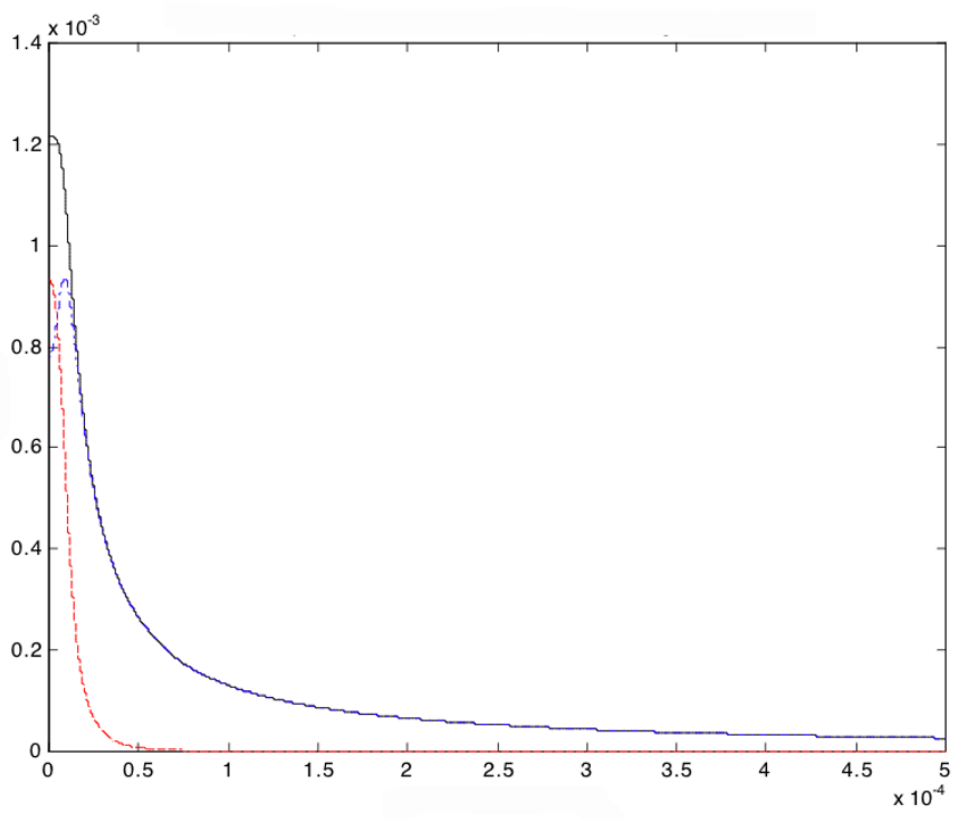

$b\left[\mathrm{~m}^{-1}\right]$

Fig. 11. Similar to Fig. 9 but the period is $600 \mathrm{~s}$.

- "Central Finland", 5 layers, (Viljanen and Pirjola, 1994)

layer thicknesses $=[12,22,16,50, \infty] \mathrm{km}$

layer resistivities $=[30000,3000,50,1000,5000]$

$\Omega \mathrm{m}$

- "Nova Scotia", 3 layers,

layer thicknesses $=[4,196, \infty] \mathrm{km}$ layer resistivities $=[0.3,10,0.3] \Omega \mathrm{m}$

Sometimes (but not in this paper) the "Central Finland" model is also assumed to include a highly-conducting bottom of $1 \Omega \mathrm{m}$ starting at the depth of $150 \mathrm{~km}$ (e.g. Pirjola et al., 2000). The "British Columbia" model is used in Figs. 9 and 11 as well but the periods $T$ equal $1 \mathrm{~s}$ and $600 \mathrm{~s}$, respec- 
tively.

In all Figs. 6-11, the real and imaginary parts of $Z_{\mathrm{w}}(b)$ are non-negative, which can also be concluded analytically from Eq. (15) for a uniform Earth (for $\omega>0$ ). Based on Eq. (9), this means that the phase of $-E_{y}$ is ahead of that of $B_{x}$ by an angle in the range from zero to 90 degrees. It is seen that the imaginary part of $Z_{\mathrm{w}}(b)$ almost always clearly exceeds the real part, especially at large values of $b$. This indicates an almost 90-degree phase difference between the electric and magnetic fields. When using the highly-conducting "Nova Scotia" model (Fig. 7) the difference between $\operatorname{Re}\left(Z_{\mathrm{w}}(b)\right)$ and $\operatorname{Im}\left(Z_{\mathrm{w}}(b)\right)$ generally seems to be smaller than in the other examples. For "Quebec" (Fig. 8) and "British Columbia" (Fig. 10), $\operatorname{Re}\left(Z_{\mathrm{w}}(b)\right)$ and $\operatorname{Im}\left(Z_{\mathrm{w}}(b)\right)$ are practically equal at $b=0$ whereas for "Central Finland" (Fig. 6), there is a clear difference even at $b=0$. In fact, the only situation in the examples where $\operatorname{Re}\left(Z_{\mathrm{w}}(b)\right)$ exceeds $\operatorname{Im}\left(Z_{\mathrm{w}}(b)\right)$ is encountered for "British Columbia" at a long period $T=600 \mathrm{~s}$ and at small $b$ values (Fig. 11).

Roughly speaking, the decay of $Z_{\mathrm{w}}(b)$ with $b$ is similar for "Central Finland" (Fig. 6) and "Quebec" (Fig. 8). For "British Columbia" (Fig. 10) the decay is somewhat slower, which obviously results from the fact that "Central Finland" and "Quebec" are more resistive than "British Columbia". To see the different decay rates, the different $b$ scale in Fig. 10 compared to Figs. 6 and 8 must be noted. The absolute value of $Z_{\mathrm{w}}(b)$ has a clear maximum at a nonzero value of $b$ for the "British Columbia" model (Fig. 10), and a maximum at $b \neq 0$ may also be seen in the "Central Finland" case (Fig. 6).

Figure 7 indicates that a highly-conducting Earth makes the variation of $Z_{\mathrm{w}}(b)$ with $b$ small. Comparing Figs. 9, 10 and 11 shows that a decrease of the period also reduces the variation of $Z_{\mathrm{w}}(b)$ with $b$. Thus, for large Earth conductivities and short periods, the dependence of $Z_{\mathrm{w}}(b)$ on $b$ is small making $Z_{\mathrm{w}}(x)$ resemble the delta function, and the plane wave approximation becomes more acceptable as discussed above in Sections 2.2 and 2.5.

\section{Discussion and Concluding Remarks}

The surface impedance expresses the relation between the horizontal geoelectric and geomagnetic fields at the Earth's surface being in general a $2 \times 2$ tensor. However, in many cases including this paper, the surface impedance is a scalar defined to equal the ratio of a horizontal electric component to the perpendicular horizontal magnetic component at the Earth's surface. Similarly to the electric and magnetic fields, the surface impedance primarily depends on time variations of the magnetospheric and ionospheric current system and is secondarily affected by currents and charges induced in the Earth. Most applications concern the latter dependence, and the surface impedance and other parameters derived from it are commonly used to characterise the Earth's conductivity structure. This kind of geomagnetic induction and magnetotelluric studies are well-known and have already been carried out for a long time all over the world.

In this paper we consider the surface impedance from a different point of view referring to space weather effects at the Earth's surface, i.e. to geomagnetically induced currents (GIC) in ground-based technological systems. The horizontal geoelectric field drives GIC and is thus the key quantity. In a usual situation in practice, geomagnetic data are available and we need to calculate the geoelectric field in order to be able to estimate GIC in a network. Knowing the surface impedance thus enables the determination of the electric field leading to a solution of the practical problem.

Since the electric and magnetic fields at the Earth's surface are functions of two space coordinates and time the surface impedance can be defined in the same domains or from field components Fourier transformed from the space coordinates to the wavenumbers and/or from the time to the frequency. The common convention is to use Fourier transformed quantities (which is an assumption in this paper, too, except for a short discussion where field components dependent on a space coordinate are applied to defining the surface impedance that is thus a function of the frequency and a space coordinate). Inverse Fourier transforming the equation that gives the electric field as the product of the surface impedance and the magnetic field in the frequency or wavenumber domain results in convolution integrals in the time or space domains. These relations are summarised in this paper. For simplicity, the discussion is restricted to one space coordinate and wavenumber.

We pay special attention to the relations of the electric field with the magnetic field and with its time derivative, which may be concluded from the surface impedance in the frequency domain. Numerical examples show that the absolute value of the surface impedance usually increases with the frequency indicating a high-pass filter character whereas the corresponding transfer functions between the electric field and the magnetic time derivative are of a low-pass filter type. However, the relative change of the latter transfer function with frequency is smaller than of the surface impedance, so in the time domain, the electric field should have more similarities with the magnetic time derivative than with the magnetic field. An artificial Earth conductivity model is also demonstrated that leads to a surface impedance which does not change much with the frequency indicating a rough proportionality between the electric and magnetic fields in the time domain. Observational evidence exists both for a proportionality between the electric and magnetic fields and for a close relation between the electric field and the magnetic time derivative. These issues constitute a subject that needs more research in the future.

The absolute value of the space-dependent surface impedance can be either larger or smaller than the absolute value of the plane wave surface impedance showing that the statement sometimes expressed that the plane wave case would give the largest electric fields is not true. A numerical example shows that, as expected, the surface impedance approaches the plane wave value at large distances from the ionospheric source current.

A discussion of a wavenumber-dependent surface impedance included in this paper indicates that in some space-dependent situations it is possible to apply the plane wave relation between surface electric and magnetic fields locally. Numerical examples particularly confirm that an increase of the Earth's conductivity or of the frequency im- 
proves the validity of the plane wave approximation. This conclusion agrees with the well-known fact in geoelectromagnetics that source effects distorting the plane wave assumption are more serious at long periods and in resistive areas.

Numerical calculations show that the absolute value of the surface impedance decreases with an increasing wavenumber although the maximum may also be achieved at a non-zero value of the wavenumber. It is seen that the imaginary part of the surface impedance usually much exceeds the real part, both of which are positive (for positive frequencies).

An assumption of a uniform Earth is a rough idealisation but it enables closed-form equations and thus makes physical interpretations easier. In this paper, we explicitly give the plane wave relation between the electric field and the magnetic time derivative in the time domain and see that the former depends on all past values of the latter with a decreasing weight. The dependence of the surface impedance on the wavenumber including an expression for the corresponding convolution kernel is also investigated under the uniform-Earth approximation.

Acknowledgments. The authors are grateful to Ari Viljanen and Antti Pulkkinen for many discussions on the subject of the paper. The authors wish to thank Mioara Mandea and an anonymous referee for critically reading the original manuscript and for many valuable and constructive comments. A revision of the paper following the referees' suggestions has definitely improved it.

\section{References}

Albertson, V. D. and J. A. Van Baelen, Electric and Magnetic Fields at the Earth's Surface Due to Auroral Currents, IEEE Trans. Power Appar. Syst., PAS-89(4), 578-584, 1970.

Avdeev, D. B., E. B. Fainberg, and B. Sh. Singer, On applicability of the Tikhonov-Cagniard magnetotelluric model for sounding of a nonuniform medium, Phys. Earth Planet. Inter., 53(3-4), 343-349, 1989.

Boteler, D. H., Geomagnetically induced currents: Present knowledge and future research, IEEE Trans. Power Delivery, 9(1), 50-58, 1994.

Boteler, D. H. and R. J. Pirjola, The complex-image method for calculating the magnetic and electric fields produced at the surface of the Earth by the auroral electrojet, Geophys. J. Int., 132(1), 31-40, 1998.

Boteler, D. H., R. J. Pirjola, and H. Nevanlinna, The effects of geomagnetic disturbances on electrical systems at the earth's surface, Adv. Space Res., 22(1), 17-27, 1998.

Cagniard, L., Basic theory of the magnetotelluric method of geophysical prospecting, Geophysics, 18, 605-635, 1953.

Dmitriev, V. I. and M. N. Berdichevsky, The Fundamental Model of Magnetotelluric Sounding, Proc. IEEE, 67, 1034-1044, 1979.

Dmitriev, V. I. and M. N. Berdichevsky, A Generalized Impedance Model, Izvestiya, Phys. Solid Earth, 38(10), 897-903, 2002.

Häkkinen, L., R. Pirjola, and C. Sucksdorff, EISCAT Magnetometer Cross and Theoret-ical Studies Connected with the Electrojet Current System, Geophysica, 25(1-2), 123-134, 1989.

Johansen, H. K. and K. Sørensen, Fast Hankel Transforms, Geophys. Prospect., 27, 876-901, 1979.

Kaufman, A. A. and G. V. Keller, The Magnetotelluric Sounding Method, Methods in Geochemistry and Geophysics, 15, Elsevier Scientific Publishing Company, 595 pp., 1981.

Lanzerotti, L. J., D. J. Thomson, and C. G. Maclennan, Engineering issues in space weather, in Modern Radio Science 1999, edited by M. A. Stuchly, 25-50, Int. Union Radio Sci. (URSI), Oxford University Press, 1999.

Lehtinen, M. and R. Pirjola, Currents produced in earthed conductor networks by geomagnetically-induced electric fields, Ann. Geophys., 3(4), 479-484, 1985.

Mareschal, M., Modelling of natural sources of magnetospheric origin in the interpretation of regional induction studies: a review, Surv. Geophys., 8, 261-300, 1986.

Osipova, I. L., S. E. Hjelt, and L. L. Vanyan, Source field problems in northern parts of the Baltic Shield, Phys. Earth Planet. Inter., 53(3-4), 337-342, 1989.

Pirjola, R., Electromagnetic induction in the earth by a plane wave or by fields of line currents harmonic in time and space, Geophysica, 18(1-2), 1-161, 1982.

Pirjola, R., Electromagnetic induction in the earth by an electrojet current system harmonic in time and space, Geophysica, 21(2), 145-159, 1985.

Pirjola, R. and D. Boteler, Calculation methods of the electric and magnetic fields at the Earth's surface produced by a line current, Radio Sci., 37(3), doi:10.1029/2001RS002576, 14-1-14-9, 2002.

Pirjola, R., A. Viljanen, and D. Boteler, Series expansions for the electric and magnetic fields produced by a line or sheet current source above a layered Earth, Radio Sci., 34(2), 269-280, 1999.

Pirjola, R., A. Viljanen, A. Pulkkinen, and O. Amm, Space Weather Risk in Power Systems and Pipelines, Physics and Chemistry of the Earth, Part C: Sol.-Terr. Planet. Sci., 25(4), 333-337, 2000.

Pirjola, R., A. Viljanen, A. Pulkkinen, S. Kilpua, and O. Amm, Space weather effects on electric power transmission grids and pipelines, Effects of Space Weather on Technology Infrastructure, NATO Advanced Research Workshop on "Effects of Space Weather on Technology Infrastructure (ESPRIT)”, Rhodes, Greece, March 25-29, 2003, edited by I. A. Daglis, NATO Science Series, Kluwer Academic Publishers, II. Mathematics, Physics and Chemistry-176, Chapter 13 "Ground Effects of Space Weather", 235-256, 2004.

Pulkkinen, A., R. Pirjola, D. Boteler, A. Viljanen, and I. Yegorov, Modelling of space weather effects on pipelines, J. Appl. Geophys., 48(4), 233-256, 2001.

Schmucker, U., Anomalies of geomagnetic variations in the southwestern United States, Bulletin of the Scripps Institution of Oceanography of the University of California, 13, La Jolla, California, USA, University of California Press, 165 pp., 1970a.

Schmucker, U., An Introduction to Induction Anomalies, J. Geomag. Geoelectr., 22(1-2), 9-33, 1970b.

Schmucker, U. and J. Jankowski, Geomagnetic induction studies and the electrical state of the upper mantle, Tectonophys., 13(1-4), 233-256, 1972.

Sokolova, E. Yu., I. M. Varentsov, and BEAR Working Group, Deep array electromagnetic sounding on the Baltic Shield: External excitation model and implications for upper mantle conductivity studies, Tectonophys., 445, 3-25, 2007.

Thomson, D. J. and J. T. Weaver, The Complex Image Approximation for Induction in a Multilayered Earth, J. Geophys. Res., 80(1), 123-129, 1975.

Trichtchenko, L. and D. H. Boteler, Effects of recent geomagnetic storms on power systems, Proceedings of the 7-th International Symposium on Electromagnetic Compatibility and Electromagnetic Ecology, SaintPetersburg, Russia, June 26-29, 2007, 265-268, 2007.

Varentsov, Iv. M., E. Yu. Sokolova, and the BEAR Working Group, Diagnostics and Suppression of Auroral Distortions in the Transfer Operators of the Electromagnetic Field in the BEAR Experiment, Isvetiya, Phys. Solid Earth, 39(4), 283-307, 2003.

Viljanen, A. and R. Pirjola, On the possibility of performing studies on the geoelectric field and ionospheric currents using induction in power systems, J. Atmos. Terr. Phys., 56(11), 1483-1491, 1994.

Viljanen, A., R. Pirjola, and L. Häkinen, An Attempt to Reduce Induction Source Effects at High Latitudes, J. Geomag. Geoelectr., 45(9), 817$831,1993$.

Viljanen, A., A. Pulkkinen, O. Amm, R. Pirjola, T. Korja, and BEAR Working Goup, Fast computation of the geoelectric field using the method of elementary current systems and planar Earth models, Ann. Geophys., 22(1), 101-113, 2004.

Wait, J. R., On the Relation between Telluric Currents and the Earth's Magnetic Field, Geophysics, 19, 281-289, 1954.

Wait, J. R., Electromagnetic surface impedance for a layered earth for general excitation, Radio Sci., 15(1), 129-134, 1980.

Wait, J. R., Wave Propagation Theory, 348 pp., Pergamon Press, New York, 1981.

Weaver, J. T., Induction in a layered plane earth by uniform and nonuniform source fields, Phys. Earth Planet. Inter., 7, 266-281, 1973.

Weaver, J. T. and A. K. Agarwal, Automatic 1-D inversion of magnetotellurtic data by the method of modelling, Geophys. J. Int., 112, 115-123, 1993.

Weidelt, P., The Inverse Problem of Geomagnetic Induction, Zeitschrift Geophys., 38, 257-289, 1972.

R. Pirjola (e-mail: risto.pirjola@fmi.fi), D. Boteler, and L. Trichtchenko 